\title{
Alerce (Fitzroya cupressoides) como Monumento Natural: un factor de desarrollo económico en la X Región de Los Lagos, Chile
}

\author{
Alerce (Fitzroya cupressoides) as Natural Monument: \\ an economic factor of development in the Lake district, Chile
}

JORGE GAYOSO, BASTIENNE SCHLEGEL

Instituto de Manejo Forestal, Universidad Austral de Chile, Casilla 567, Valdivia, Chile.

SUMMARY

This paper analyses the possibilities of improving the economic development of the Lake Region in Chile through a tourism proposal associated to alerce as a natural monument. Alerce, the natural patrimony object of this study is described and analysed, characterising the current demand and its trends. Finally, some points of view about the value of the resource are commented and the advantages and disadvantages of a possible tourist development associated to the natural areas where alerce grows are discussed. The Natural Monument Alerce is an attractive resource, among others, for its scenic beauty, its uniqueness and the millennial longevity of its individuals. In consequence, the economy associated to this resource, product of the demand of the new tourist destinations to pristine places which consider the environment, can be considered a suitable instrument for local development.

Key words: Fitzroya cupressoides, ecotourism, economic development.

\section{RESUMEN}

Este documento analiza las posibilidades de incrementar el desarrollo económico en la X Región de Los Lagos, Chile, a través de una propuesta de un turismo asociado al patrimonio denominado Monumento Natural Alerce. Luego de introducir al tema, se describe el patrimonio natural objeto del estudio, se analiza la oferta actual y las posibilidades de ampliarla, y caracteriza la demanda actual y sus tendencias. Finalmente, se comentan algunos puntos de vista sobre la valorización del recurso y discute las ventajas y desventajas de un posible desarrollo turístico asociado a estas áreas naturales donde crece alerce. El Monumento Natural Alerce es un recurso atractivo, entre otros, por su belleza escénica, su carácter único y la longevidad milenaria de sus individuos. En consecuencia, la economía asociada a este recurso, producto de la demanda de los nuevos destinos turísticos hacia lugares prístinos que incorporan el entorno ambiental, puede considerarse un adecuado instrumento de desarrollo local.

Palabras claves: Fitzroya cupressoides, ecoturismo, desarrollo económico.

\section{INTRODUCCION}

En los estudios relacionados con el desarrollo económico está siendo considerada cada vez con más relevancia la economía de la cultura y el patrimonio (Herrero 1997). Esto se debe, según el mismo autor, al comportamiento de la sociedad en cuanto al aumento del consumo de este tipo de productos y los efectos económicos que puede generar el sector cultural, en términos de empleo, ingresos, producción, y su implicancia en las es- trategias de desarrollo económico de las ciudades y regiones.

Existe una creciente demanda de la sociedad actual por los distintos usos del tiempo libre, entre ellos los destinos turísticos de carácter natural y en especial por el denominado turismo alternativo, que incluye al turismo de intereses especiales, de aventura y ecoturismo. Este nuevo mercado aparece como consecuencia de la saturación de destinos turísticos tradicionales. Ello posibilita una revalorización de los destinos naturales y ofrece una 
nueva oportunidad de desarrollo a las localidades cercanas a los sitios de mayor belleza escénica. Adicionalmente, el turismo de ambientes naturales es una alternativa estratégica que puede colaborar a preservar el patrimonio ambiental del país al poner en valor sitios de interés por su belleza natural, evitando así la presión sobre el paisaje de actividades competitivas de carácter extractivo: diversificar y ampliar áreas potencialmente aprovechables, asignarle valor social y económico al medio ambiente, disponer recursos para ser destinados a la protección y conservación del patrimonio natural, incrementar la participación de la población local, generar empleos, favorecer e incentivar pequeñas empresas de artesanía, alimentos y otros productos y servicios destinados a los visitantes (Rivas y Villarroel 1995).

Los ambientes naturales poco intervenidos, silvestres, prístinos, son un destino cada vez más valorado por los turistas internacionales. Así, el turismo en espacios naturales representa una oportunidad significativa de desarrollo económico para algunas regiones de Chile, en especial aquellas con mayor índice de ruralidad y las más pobres. Además, si este desarrollo se planifica, de modo que sea ambientalmente sustentable, la oportunidad señalada tiene la ventaja de ser conservativa para el patrimonio natural y la biodiversidad contenida en él (Rivas y Villarroel 1995).

En este marco de referencia, se propone incrementar la oferta de servicios asociados al Monumento Natural Alerce, que comprende la conservación de la segunda especie más longeva del planeta y endémica del sur de Chile y Argentina.

\section{EL MONUMENTO NATURAL ALERCE}

Alerce, Fitzroya cupressoides, es una especie endémica del centro-sur de Chile y algunos sectores de Argentina. En Chile crece como poblaciones discontinuas en la Décima Región, desde $39^{\circ}$ $50^{\prime}$ hasta $42^{\circ} 30^{\prime} \mathrm{S}$ en la Cordillera de la Costa y desde $41^{\circ}$ hasta $43^{\circ} 30^{\prime} \mathrm{S}$ en los Andes (Donoso 1981); además, se encuentra escasamente en la depresión intermedia en las cercanías de Puerto Montt. Estudios dendrocronológicos han demostrado que alerce es la segunda especie más longeva a nivel mundial y puede alcanzar hasta 5 metros de diámetro, 50 metros en altura y edades superiores a los 3600 años (Lara y Villalba 1993). Los alerces son testigos milenarios del clima planeta- rio; investigaciones en la especie han permitido hacer reconstrucciones de temperatura para los últimos 3620 años (Lara et al., 1994).

El alerce fue una de las primeras especies forestales explotadas comercialmente en Chile (Donoso et al. 1990). El gran interés en la explotación del alerce fue motivado por las características de su hermosa madera color rojizo-café con un fino vetado, la estabilidad dimensional, durabilidad y gran resistencia a los agentes climáticos. La madera de alerce se utilizó en toda clase de terminaciones interiores y fue extensamente utilizada en cubiertas y revestimientos exteriores como tejuela. De acuerdo con historiadores, en el pasado, tanto en Llanquihue como en Chiloé, las tablas de alerce fueron aceptadas como moneda de pago y aun llegó a recibir un trato similar en el puerto de Valparaíso (Reiche 1934, citado por Veblen et al. 1976).

La explotación del alerce representa una parte de la historia cultural de Chile debido a que ninguna otra especie ha sido tan explotada a través de los siglos. La explotación de alerce empezó en el período de la colonización española. Hay evidencias de embarques de madera de alerce desde la isla de Chiloé ya en 1641 (Fonck 1896, citado por Donoso et al. 1990). En 1644 se relata que aparte de la producción de textiles, la producción de tablones de alerce era la industria más importante de Chiloé. El archipiélago de Chiloé se convirtió en un importante proveedor de madera para Chile y particularmente para Lima (Ramírez 1996). Hasta mediados del siglo XIX la madera de alerce fue el principal producto de exportación de Chiloé.

La explotación de alerce continuó a través de la historia al punto que a fines del siglo XIX los bosques accesibles cercanos a Puerto Montt y al estuario de Reloncaví habían sido cortados y prácticamente eliminados (Veblen et al. 1976). Muchos de estos bosques fueron quemados para habilitar tierras para la agricultura, como consecuencia de la colonización alemana (Veblen y Ashton 1982). La continua explotación de alerce y destrucción de su hábitat, debido a la belleza y durabilidad de su madera, han restringido la distribución natural de la especie, siendo hoy clasificada como una especie amenazada y protegida por varias leyes (Lara 1991). Según CONAF et al. (1997) el alerce está presente en Chile, aún en 264993 hectáreas ubicadas tanto en la Cordillera de los Andes como en la Cordillera de la Costa y 
algunos sectores de la depresión intermedia cercanos a Puerto Montt, de las cuales 47395 ha están dentro del SNASPE. La drástica reducción de los bosques de alerce impulsó a Argentina (1973) y luego Chile (1975) a incluir a esta especie en el Apéndice I (Prohibición absoluta de todo comercio internacional) de la Convención sobre Comercio Internacional de Especies Silvestres de Flora y Fauna en vías de Extinción (CITES).

El $1^{\circ}$ de octubre de 1976 el Ministerio de Agricultura de Chile dictó el decreto $\mathrm{N}^{\circ} 490$, el cual declara Monumento Natural al alerce vivo, de acuerdo a la Convención para la Protección de la Flora, Fauna y Bellezas Escénicas Naturales de América. Su redacción contiene principios ecológicos fundamentales, haciendo hincapié en las razones que ameritan la protección de esta especie: alerce es una especie rara de gran valor científico e importancia cultural; es una de las especies más longevas del reino vegetal; que ha sido objeto en las últimas décadas de una explotación intensa e irracional, la que de continuar significará, a breve plazo, la extinción de los últimos bosques de alerce; que la presencia del alerce constituye un extraordinario atractivo y recurso botánico científico y turístico de renombre internacional que se hace necesario conservar (Castro 1995).

Este decreto en su artículo segundo declara: "A partir de la fecha de publicación en el Diario Oficial del presente decreto, declárase inviolable y prohíbese la corta y destrucción del alerce, salvo autorización expresa, calificada y fundamentada de la Corporación Nacional Forestal..." La publicación en el Diario Oficial se hizo el 5 de septiembre de 1977.

Los párrafos siguientes corresponden al análisis tradicional de comportamiento de la oferta y demanda: los bienes que existen y su valor, cómo se expresa la demanda, con qué intensidad y cómo se puede influir en el mercado.

\section{CARACTERIZACION DE LA OFERTA. ZONAS CON ALERCE INCLUIDAS \\ EN EL SISTEMA NACIONAL DE AREAS SILVESTRES PROTEGIDAS DEL ESTADO (SNASPE)}

Chile es uno de los apenas cinco países con ecosistemas de latitud sur extremos junto a Sudáfrica, Nueva Zelanda, Australia y Argentina. Tiene los bosques templados más australes del planeta, campos de glaciares y fiordos. El SNASPE incluye actualmente 32 parques nacionales, 43 reservas nacionales y 12 monumentos naturales, los que ocupan alrededor de 14.6 millones de hectáreas.

La oferta está constituida por las áreas protegidas del Estado y Parques Privados conteniendo bosques de alerce. Las primeras se iniciaron en 1982, creándose varios Parques Nacionales y una Reserva Forestal (cuadro 1) y las segundas son más recientes (cuadro 2).

\section{CUADRO 1}

Areas del SNASPE con alerce.

SNASPE areas with alerce.

\begin{tabular}{|l|c|c|}
\hline Tipo de Zona Protegida & Area total (ha) & Con alerce (ha) \\
\hline Parque Nacional Alerce Andino & 39255 & 79186 \\
Parque Nacional Chiloé & 43057 & 9741 \\
Parque Nacional Hornopirén (1988) & 48232 & 4148 \\
Reserva Forestal Llanquihue & 33972 & 1341 \\
Monumento Natural Alerce Costero & 2308 & 41676 \\
\hline Total & 166824 & \multirow{2}{*}{} \\
\hline
\end{tabular}

Fuente: INFOR et al. (1995). 
Los bosques de alerce contienen una importante variedad de fauna y flora, incluyendo ejemplares milenarios de alerce. Su buen estado de conservación en el Monumento Nacional Alerce Costero es el principal valor y potencial en la Cordillera de la Costa. Allí se pueden apreciar especies como coigüe, canelo, tineo, trevo, mañío hembra y macho, tepa, ulmo, olivillo y desde luego alerce. Entre la fauna de mamíferos destacan los pumas, zorros, guiñas, hurones, monitos del monte y chingues. Las aves más avistadas son el halcón, buitre, aguilucho y el chucao (Ambiente y Desarrollo 1995).

Estas áreas bajo el SNASPE representan alrededor del $17 \%$ de la superficie con bosques de alerce en Chile, por lo que la oferta podría incrementarse. Sin embargo, el principal problema de la oferta actual es la carencia de infraestructura vial de acceso adecuada y falta de una oferta de servicios asociados para atender la creciente y sostenida demanda de visitantes. También hay algunas iniciativas privadas para llevar turistas ofreciendo una hermosa experiencia con la naturaleza y favoreciendo el desarrollo local, pero están aún lejos de considerarse apropiadas y de calidad para atraer capacidad de gasto y oportunidades reales de desarrollo económico.

Chile, además, gasta poco en promoción, 0.7 US\$ por cada turista ingresado. En el tema de la promoción y la consideración del aumento de las exigencias y expectativas de los visitantes en cuanto a la calidad de servicios recreativos, de alojamiento y otros, el Estado a través de la Corporación Nacional Forestal (CONAF) desde 1996 ha iniciado una estrategia de difusión de oportunidades de ecoturismo y turismo aventura y un programa para la gestión de uso público en las áreas del SNASPE (Lazo 1996). Según la Organización Mundial de Turismo, el ecoturismo y turismo aventura están creciendo a una tasa del $30 \%$ a diferencia del convencional que lo hace a $7 \%$ anual, buena razón para incentivar la promoción. La promoción, de responsabilidad de la Administración Pública, es una de las esferas de acción que propone Herrero (1997) con la finalidad de desarrollar un sentimiento de pertenencia e identificación de los ciudadanos con sus recursos, y por otra, posicionar la oferta en el contexto nacional y extranjero.

CONAF por gestión directa no ha podido revertir la situación de carencias y los recursos aportados por el Estado no son suficientes. Actualmente se invierten US\$ 5.2 millones en las áreas del SNASPE, de los cuales el $17 \%$ va a manteni- miento y $13 \%$ a inversión. Por ello, adquiere tanta importancia la estrategia de hacer partícipe al sector privado, mediante el mecanismo de concesiones de la gestión de mejoramiento de la oferta de servicios recreativo turísticos. Los derechos de concesión se reinvertirían para consolidar la infraestructura y equipamiento para protección, educación, interpretación ambiental y apoyo a la investigación.

Como una forma de hacer crecer la oferta de las áreas protegidas y favorecer su continuidad, el Estado chileno está tratando de incentivar el interés privado en la adquisición de superficies. Actualmente la Comisión Nacional de Medio Ambiente (CONAMA), la CONAF y el Ministerio de Hacienda están trabajando en la elaboración de una ley especial para incentivar la creación de Areas Silvestres Protegidas Privadas (ASPP). Esto a partir de una bonificación del manejo de esas áreas que cubriría los costos de elaboración y ejecución de Planes de Manejo para las ASPP. Considera además la exención del impuesto territorial (2\% del avalúo fiscal), del impuesto a la renta presunta y del impuesto global complementario (Sepúlveda 1997).

Por otra parte, la solución del problema de la oferta de servicios pasa por una planificación de largo plazo. Si bien el sector privado ha ido respondiendo a la tendencia de la demanda, su adecuación es por metas de corto plazo y principalmente reactiva. Es decir, el desarrollo del sector tiene un carácter espontáneo y por lo cual presenta los riesgos de posible sobresaturación de los sitios de mayor demanda, pérdida de activos turísticos potenciales o futuros y cierta tendencia a reproducir patrones de desarrollo turístico externo.

La oferta de servicios puede comprender, por ejemplo, el desarrollo de un museo del alerce (piezas petrificadas, dendrocronología, interpretación histórica de su explotación, usos), tal vez asociado a la cultura del pleistoceno tardío descubierta en los sitios de Monte Verde (Puerto Montt) y fechados en 12500 y 33000 años de antigüedad, lo cual ha hecho cambiar las ideas de la llegada del Homosapiens a esta parte del cono sur de América (Pino 1998). Este hallazgo, que contempla piezas de madera elaboradas por el hombre, ya reconocido por la comunidad científica internacional, transformará el sitio en una atracción mundial. Eventos culturales y científicos, circuitos hacia alerces milenarios, desarrollo de artesanías y producción de bienes como libros y videos, pueden complementar la oferta. 


\section{CUADRO 2}

Parques y Reservas Privadas con alerce.

Private Parks and Reserves with alerce.

\begin{tabular}{|c|c|c|}
\hline Nombre & Localización & Descripción \\
\hline $\begin{array}{l}\text { Santuario de } \\
\text { la Naturaleza } \\
\text { Pumalín }\end{array}$ & $\begin{array}{l}\text { Palena } \\
\text { X Región }\end{array}$ & $\begin{array}{l}251519 \text { ha en la provincia de Palena adquiridas a partir de } 1991 \text { a la } \\
\text { fecha, de propiedad de Douglas Tompkins ( } 4068 \text { ha) y de El Bosque } \\
\text { Pumalín Foundation ( } 247491 \text { ha). Se está solicitando su declaración } \\
\text { como Santuario de la Naturaleza por el Consejo de Monumentos Na- } \\
\text { cionales. Se contempla su traspaso a la Fundación EDUCEC. Se rea- } \\
\text { lizan programas de educación, recreación e investigación ecológica, y } \\
\text { se implementan proyectos de desarrollo rural basado en la conserva- } \\
\text { ción, a través de predios demostrativos y transferencia tecnológica. La } \\
\text { inversión total hasta la fecha es de } 15 \text { millones de US\$. }\end{array}$ \\
\hline $\begin{array}{l}\text { Parque } \\
\text { Ayacara }\end{array}$ & $\begin{array}{l}\text { Palena } \\
\text { X Región }\end{array}$ & $\begin{array}{l}\text { Aproximadamente } 12 \text { mil hectáreas adquiridas por la familia Sandor } \\
\text { en } 1978 \text { con fines de conservación. Gradualmente, estas tierras se han } \\
\text { ido convirtiendo en un Parque Privado abierto al uso público y en un } \\
\text { programa más amplio de educación, investigación y acción dirigido } \\
\text { principalmente hacia la comunidad de Ayacara. En } 1990 \text { se construyó } \\
\text { un hotel, y en } 1993 \text { se creó la Fundación Ayacara. }\end{array}$ \\
\hline $\begin{array}{l}\text { Parque del } \\
\text { Estuario SA, } \\
\text { sector } \\
\text { Factoría }\end{array}$ & $\begin{array}{l}\text { Cochamó- } \\
\text { Canutillar } \\
\text { X Región }\end{array}$ & $\begin{array}{l}\text { Consiste en la protección de un área silvestre en una superficie total de } \\
1950 \text { ha } 10 \mathrm{~km} \text { al sur de Canutillar -adquiridas en } 1995 \text { por un aviso } \\
\text { en el diario (US\$ } 150 \text { mil)-, de las cuales un } 90 \% \text { se dedica a conser- } \\
\text { vación y el resto se destina a multiuso con viviendas e infraestructura } \\
\text { para el uso recreativo. Funciona como una sociedad con } 43 \text { accionistas } \\
\text { (la meta son 50) en lo patrimonial, y a la vez como una corporación } \\
\text { privada para el uso y goce, que se encuentra reglamentado. Se han } \\
\text { realizado dos estudios: un catastro de especies, ecosistemas y unidades } \\
\text { fisiográficas (con levantamiento topográfico) y uno histórico y social } \\
\text { del sector. La expectativa es generar un parque privado abierto a pú- } \\
\text { blico a través de convenios con universidades (investigación) y cole- } \\
\text { gios (educación). El } 90 \% \text { del parque limita con el PN Alerce Andino. } \\
\text { No se han vinculado con organismos públicos. El parque podría ser un } \\
\text { acceso oriente al PN Alerce Andino en una zona que no tiene acceso. } \\
\text { Tienen Plan de Manejo. La filosofía es que no sea una conservación } \\
\text { estática sino buscar una relación natural entre ser humano y medio } \\
\text { ambiente. }\end{array}$ \\
\hline $\begin{array}{l}\text { Alerce } \\
\text { Mountain } \\
\text { Lodge }\end{array}$ & $\begin{array}{l}\text { Lenca } \\
\text { X Región }\end{array}$ & $\begin{array}{l}2 \text { mil ha adquiridas en } 1995 \text { con el fin de explotarlas madereramente, } \\
\text { en especial el alerce. Por su carácter único en cuanto a los bosques que } \\
\text { contiene y a su ubicación (colinda con el Parque Alerce Andino) el } \\
\text { dueño decidió proteger una parte y dedicarla al ecoturismo. Construyó } \\
\text { un hotel y cabañas, un camino que llega hasta la parte más alta y en } \\
\text { noviembre de } 1997 \text { la abrió al público. Actualmente sólo trabaja el } \\
\text { alerce muerto en la mitad del campo. En el resto no hay explotación. } \\
\text { El bosque "verde", de maderas blancas, no se explota en ninguna zona } \\
\text { del fundo. }\end{array}$ \\
\hline
\end{tabular}

Fuente: Sepúlveda et al. (1998). 


\section{VALOR ECONOMICO DEL MONUMENTO NATURAL ALERCE}

Existen distintos métodos para la determinación de la demanda de servicios recreativos de los espacios naturales (Azqueta y Pérez 1996). Conocer el valor que les otorga la sociedad y el beneficio que los individuos obtienen de la existencia de esas áreas, es útil para la toma de decisiones como inversión en su conservación y mantenimiento, recuperación de entornos degradados, jerarquización de usos alternativos excluyentes, etc. Entre los métodos para derivar la función de demanda, se tiene el método del costo de viaje y la valoración contingente.

Siguiendo a Sánchez y Donoso (1996) y Sepúlveda (1997), el valor económico total de un recurso natural como el Monumento Natural Alerce puede ser medido como el valor presente descontado de la suma de todos los servicios que provee a las personas. Ya sea por el beneficio de uso directo (extractivos y no extractivos como la recreación), los valores de uso indirecto (regulación ecológica a través de la captura de dióxido de carbono, protección de suelos, regulación hídrica), no uso de dicho recurso (valor de opción o legado, valor intrínseco o de existencia, altruismo) y bienes y servicios complementarios al recurso. Los bienes complementarios serían los servicios de viaje hacia las áreas, los eventos y otros bienes como son la representación visual o literaria del recurso, a través de libros, videos u otros que no significan una utilización in situ, pero que están vinculados con el patrimonio natural.

Por lo tanto, resulta difícil revelar la demanda de un patrimonio natural, ya que de acuerdo con Herrero (1997) ésta se manifiesta de forma colectiva como en todo bien público. Así, los precios de estos bienes en forma de las entradas y tickets de visita no reflejan la real escasez del bien demandado, puesto que en su mayor parte son precios subvencionados y no reflejan el costo de conservación o de mantenimiento. Una particularidad de la demanda de este tipo de patrimonio es que los individuos no buscan el bien en particular, sino los componentes de valor que lleva incorporado y por lo tanto los servicios que pueden derivarse. Incorporando el análisis de Herrero et al. (1998), cuando se visita un área natural, no se demanda el bien en sí mismo, sino el conjunto de valores y servicios que están asociados, y que incluyen desde la emoción estética hasta el valor de formación y de educación. Mientras la oferta de patrimonio puede considerarse fija como stock, no lo son los servicios complementarios y es la demanda de estos últimos la que le da gran parte del valor económico al recurso natural.

A pesar que el mercado es ineficiente para revelar el valor económico total del Monumento Natural, por su condición de bien público, existen métodos para determinar el valor de existencia o valor per se basado en relación con los valores propios y que debe ser determinado en condición de no uso. Una medición posible es a través de la propiedad de sustituibilidad y que se expresa en términos de la disposición a pagar y la de disposición a aceptar, valor que es determinable por el método directo de valoración contingente.

En Chile no existen estudios que permitan estimar el valor económico total de las áreas silvestres protegidas existentes (ASP). Algunas investigaciones permiten sólo una aproximación (Sepúlveda 1997). Por valoración contingente se ha estimado, por ejemplo, que la disposición a pagar de los usuarios en seis de las unidades más visitadas de! SNASPE bordea en promedio los US\$ 300000 anuales. Este cálculo se realizó consultando a una muestra de visitantes su disposición a pagar por el valor de opción, de existencia, de legado y de altruismo. Si a la cifra anterior se suman los gastos realizados por los usuarios de las unidades estudiadas durante su visita, se tiene que los beneficios monetarios anuales en promedio para cada una de las ASP incluida la disposición a pagar podría alcanzar a US\$ 5.17 millones anuales. Esta cifra supera en casi 40 veces los US\$ 130000 que CONAF percibió como ingresos promedio totales para cada una de las áreas durante 1995. La magnitud de esta valoración estaría justificando plenamente la inversión pública en el fomento y creación de áreas silvestres protegidas en Chile, desde una perspectiva social.

\section{CARACTERIZACION DE LA DEMANDA ACTUAL Y POTENCIALIDAD}

Los antecedentes de la demanda sobre el Monumento Natural Alerce se configuran a partir de estadísticas de CONAF y del Servicio Nacional de Turismo (cuadro 3). La llegada de visitantes extranjeros a las áreas silvestres protegidas a nivel nacional ha crecido de 93600 a 169000 entre los años 1990 y 1994. De éstos, llega un $44 \%$ a las 


\section{CUADRO 3}

Visitantes a las Areas Silvestres Protegidas, Décima Región

Visitors to Protected Wild Areas, $10^{\text {th }}$ Region

\begin{tabular}{|c|c|c|c|c|c|}
\hline \multirow{2}{*}{$\begin{array}{l}\text { Areas silvestres } \\
\text { protegidas }\end{array}$} & \multicolumn{5}{|c|}{ Total visitantes por año } \\
\hline & 1990 & 1991 & 1992 & 1993 & 1994 \\
\hline MN Alerce Costero & 109 & 131 & 204 & 236 & 258 \\
\hline PN Puyehue ${ }^{1}$ & 40046 & 43637 & 52047 & 63551 & 64607 \\
\hline PN Pérez Rosales & 82212 & 82992 & 117429 & 148967 & 162130 \\
\hline PN Alerce Andino & 1216 & 2378 & 3720 & 5980 & 8168 \\
\hline RN Llanquihue & 180 & 251 & 260 & 257 & 283 \\
\hline PN Chiloé & 7207 & 10225 & 10660 & 13653 & 9661 \\
\hline RN Valdivia & s.i. & s.i. & s.i. & s.i. & 200 \\
\hline PN Hornopirén & s.i. & s.i & s.i & s.i & s.i \\
\hline Total & 130970 & 139614 & 184320 & 232644 & 245307 \\
\hline
\end{tabular}

s.i. Sin información.

Fuente: CONAF. 1992. Memoria anual CONAF; 1994-95: Datos no publicados (Comunicación personal Sr. Carlos Sarmiento, Unidad de Gestión Patrimonio Silvestre, CONAF).

1 El área identificada como Puyehue no contiene alerce.

áreas silvestres protegidas de la X Región. Además es notable la participación que tienen los extranjeros en el total de los visitantes a estas áreas, alcanzando más del 30 por ciento (SERNATUR 1995).

Según cifras de CONAF, hay un ritmo sostenido de crecimiento del turismo en áreas silvestres protegidas (5\% anual); desde 1986 a 1993 los visitantes a Parques Nacionales subieron de $661 \mathrm{mil}$ a 842 mil. En el mismo período, los visitantes extranjeros subieron de 8 a $12 \%$ del total nacional. Sin embargo, la llegada de visitantes a las áreas con recurso alerce no alcanza al $10 \%$ de los que llegan a la X Región y son sólo un dos por ciento del total de visitantes a las áreas del SNASPE en Chile, denotando claramente su menor desarrollo y dificultades de acceso.

Además del incremento de turistas extranjeros que buscan lugares prístinos, en los últimos años el consumo de patrimonio natural ha crecido de forma importante, entre otras razones, por el fortalecimiento de una clase media y la presión que surge de las grandes problemáticas ambientales en los grandes centros urbanos de Chile.
Las perspectivas de Chile como destino turístico pueden calificarse como promisorias. De acuerdo con cifras de la Organización Mundial de Turismo, Chile se encuentra entre los principales destinos turísticos en América, con una tasa de crecimiento anual promedio de $17.5 \%$ en los últimos 10 años, tasa muy superior al promedio que registra el continente americano en su conjunto. En 1994 la llegada de turistas superó 1.6 millones con un aporte estimado de US\$ 833 millones (Rivas y Villarroel 1995). El tiempo de estada promedio fue de 10 días para los turistas americanos y 16 días para los provenientes de otros países. Se estima que cerca de un quinto de los turistas que hoy ingresan a Chile lo hacen interesados en el turismo alternativo, produciendo un ingreso de cerca de US\$ 150 millones y que podría llegar a US\$ 480 millones para el año 2000 (Rivas y Villarroel 1995).

Esto pone de manifiesto la importancia del turismo y los servicios relacionados como expresión cada vez más importante del empleo del ocio por parte de la sociedad contemporánea y, por lo tanto, que la rentabilidad económica de los bienes 
patrimoniales proviene de las posibilidades de integración de estos elementos en los circuitos de consumo turístico. La apuesta de Chile debe orientarse principalmente al segmento de turistas extranjeros, dado que a pesar del mejoramiento del ingreso interno per cápita en los últimos años, por la inadecuada distribución actual del ingreso se tiene aún un bajo gasto promedio de las familias chilenas en vacaciones $(0.35 \%)$, giras turísticas $(0.10 \%)$, esparcimiento y cultura $(1.29 \%)$, recreación y educación $(7.77 \%)$, con relación a su ingreso (Törey 1996).

\section{TURISMO Y DESARROLLO ECONOMICO LOCAL}

El efecto del turismo en el desarrollo local es un aspecto poco estudiado y en general se percibe en el caso de áreas del SNASPE que las comunidades locales adyacentes no reciben los efectos positivos de esta actividad de turismo alternativo, o al menos no con la intensidad esperada.

Un ejemplo positivo estudiado es el caso del Parque Nacional Torres del Paine y su incidencia sobre la localidad próxima de Puerto Natales, donde 1 de cada 9 familias tiene en la actualidad ingresos directos provenientes del turismo en la localidad. Un $3.5 \%$ de la población económicamente activa se emplea en la actividad turística (Villarroel 1996). La valoración social del turismo por los trabajadores locales, a partir de los resultados de ese estudio, muestra que los trabajadores sienten que el turismo además del ingreso les permite capacitarse y crecer como personas, se encuentran mayoritariamente conformes con el trabajo turístico que desempeñan y la gente percibe que la actividad turística es una actividad en crecimiento en Natales. Esto ha actuado corno factor de arraigo de personas y permite ser optimistas para aplicar a otras zonas.

Sin embargo, existen algunos temores de la población local y se refieren tanto al auge que puede llevar a interesar a grandes empresas turísticas que dejarían fuera o al menos afectarían la participación de los locales, generando inestabilidad e inequidad económica. Las empresas operadoras mayoristas rara vez incorporan dentro de sus programas y servicios a la población local, especialmente las operadoras que mueven turistas extranjeros. La otra inquietud dice relación con la marcada estacionalidad de la actividad, lo que debiera llevar a una mayor creatividad para extender la época y ofrecer servicios alternativos.

El Monumento Natural Alerce podrá llegar a ser factor de desarrollo económico local, sólo en la medida que logre hacer surgir actividades económicas que den empleo e ingresos continuos, retengan a la población local y atraigan inversiones. Además, hay que buscar que el impacto sociocultural sea positivo, que el desarrollo económico no afecte sus formas de vida, tradiciones y estilos de vida. Para concretar el desarrollo no sólo debe existir demanda por este tipo de bienes, sino que se debe proporcionar una oferta adecuada, ya que oferta y demanda se potencian (Herrero et al. 1998).

\section{LIMITES DEL USO TURISTICO EN AREAS SILVESTRES PROTEGIDAS}

Considerando el vertiginoso aumento del ecoturismo y otras formas de turismo no convencional, el tema de los límites del uso turístico de las áreas silvestres protegidas aparece como una preocupación de los investigadores y administradores de las áreas (Otero y Rivas 1995). Hoy, la ausencia de planificación y estudios de capacidad de carga entre otros, sumados al desarrollo de la infraestructura, hacen temer la aparición de algunos problemas de degradación ambiental: deforestación, erosión, lanzamiento de basura, efluentes cloacales sobre cursos y cuerpos de agua, etc. (Manning 1996). En general, una alta dispersión de las áreas de acampe en las áreas silvestres protegidas imposibilita con recursos escasos su mantenimiento y control, con la consiguiente posible degradación del paisaje.

Por lo tanto, aparece la necesidad de investigación científica de los recursos naturales, sus diversas interrelaciones biogeoquímicas, diversidad y estabilidad ecosistémica, que sirva para establecer modelos biológico turísticos. A partir de esta base se proyectarían los niveles de aprovechamiento económico de las áreas menos susceptibles de impacto (Fierro 1996). El reconocimiento del patrimonio natural como una opción estratégica de desarrollo es un enorme desafío para los gobiernos regionales y empresarios, que requiere capacidad de gestión y visión de largo plazo. 


\section{CONCLUSIONES}

Del análisis anterior se puede concluir que la economía asociada al patrimonio natural está adquiriendo importancia en Chile por los efectos económicos que puede generar, comenzando a ser considerada como instrumento eficaz en las estrategias de desarrollo local y regional. Esto aparece especialmente válido para las áreas menos desarrolladas del país.

El Monumento Natural Alerce es un recurso atractivo, entre otros, por su belleza escénica, su carácter único y la longevidad milenaria de sus individuos. En consecuencia, se corresponde con la demanda de los nuevos destinos turísticos hacia lugares que incorporan el entorno ambiental, viajes más especializados, destinos prístinos. Prueba de ello la da el creciente flujo de turistas extranjeros a las áreas silvestres protegidas de Chile.

No obstante, el crecimiento de la demanda por áreas silvestres protegidas en la X Región y la existencia de una oferta suficiente de stock de recurso natural no se refleja en la demanda de las áreas con alerce. Esto se debería, principalmente, al insuficiente desarrollo de infraestructuras y ausencia de servicios complementarios en esas áreas, lo que no es correspondiente con el creciente nivel de exigencia de turistas cada vez más experimentados.

El Monumento Alerce tiene que aprovechar su condición de ecosistema único y puede transformarse en factor de desarrollo local, en cuanto haga surgir actividades económicas que generen empleo e ingresos permanentes, favorezca el arraigo de sus habitantes y atraiga inversiones.

Para lograr una efectiva conservación del Patrimonio Natural Alerce al servicio del desarrollo, es indispensable que los gobiernos regionales reconozcan sus activos naturales potenciales, demuestren la voluntad de conservarlos, y sean capaces de diseñar estrategias de mediano y largo plazo que faciliten el concurso del sector privado en esta tarea. Los privados están cada vez más interesados y dispuestos a colaborar con el sector público en iniciativas de conservación del patrimonio silvestre, por lo cual éste es un desafío relevante y posible de alcanzar.

Sin perjuicio de las acciones y responsabilidad del Estado en la fijación de las políticas y regulaciones para las áreas del SNASPE, se sugiere promover la gestión participativa en conjunto con actores vinculados al manejo del uso público, fo- mentar la capacitación y promover los programas de ciencia y tecnología, entre otros. El turismo debe ser considerado como la principal alternativa financiera para el desarrollo de las Areas Silvestres Protegidas, especialmente el caso del Monumento Natural Alerce.

\section{BIBLIOGRAFIA}

AMBIENTE Y DESARROLLO. 1995. "En Monumento Nacional Alerce Costero: Turismo ecológico potenciará conocimiento de prístina reserva de bosque valdiviano", Ambiente y Desarrollo. $\mathrm{Vol} \mathrm{XI}, \mathrm{N}^{\circ} 4: 85$.

AZQUETA, D., L. PEREZ. 1996. Gestión de espacios naturales. La demanda de servicios recreativos. McGraw Hill, Madrid, $237 \mathrm{p}$.

CASTRO, E. 1995. Erase una vez un Bosque. Mimeografiado. $187 \mathrm{p}$.

CONAF (CORPORACION NACIONAL FORESTAL), CONAMA (COMISION NACIONAL DEL MEDIO AMBIENTE), UACH (UNIVERSIDAD AUSTRAL DE CHILE), PUC (PONTIFICIA UNIVERSIDAD CATOLICA) y UCT (UNIVERSIDAD CATOLICA DE TEMUCO). 1997. Resultados finales síntesis, Catastro y Evaluación Recursos Vegetacionales Nativos de Chile.

DONOSO, C. 1981. Tipos forestales de los Bosques Nativos de Chile. Documento de Trabajo $\mathrm{N}^{\circ} 38$. Investigación y desarrollo forestal (CONAF/PNUD/FAO). Publicación FAO, Chile: 64-70.

DONOSO, C., R. GREZ, V. SANDOVAL. 1990. "Caracterización del tipo forestal alerce", Bosque 11(1): 21-34.

FIERRO, M. 1996 "Turismo tradicional, bioturismo y medio ambiente", Ambiente y Desarrollo. Vol. XII, N4: 65-69.

HERRERO, L. 1997. Economía de la cultura y el ocio. Nuevas posibilidades para la Política Económica Regional. Mercurio. Rev. de Economía y Empresa, $\mathrm{N}^{\circ}$ 1, 101-118.

HERRERO, L., E. VICENTE, M. DEVESA. 1998. El factor cultural en el desarrollo económico de las regiones desfavorecidas. El caso de la Comunidad Autónoma de Castilla y León (España). I International Scientific Congress "Tourism and Culture for Sustainable Development". Mimeo. $15 \mathrm{p}$.

INFOR (INSTITUTO NACIONAL FORESTAL), CORMA (CORPORACION CHILENA DE LA MADERA), CONAF (CORPORACION NACIONAL FORESTAL). Estadísticas Forestales X Región 1995. Valdivia, 129 p.

LARA, A. 1991. The dynamics and disturbance regimes of Fitzroya cupressoides forests in South-Central Andes of Chile, PhD Thesis Dep. of Geography of Colorado, University of Colorado.

LARA, A., R. VILLALBA. 1993. "A 3620 - year temperature record from Fitzroya cupressoides tree rings in Southern", South America. Science 260: 1104-1106.

LARA, A., J. ARAVENA, R. VILlalBA. 1994. "Alerces, testigos milenarios del clima planetario", Ambiente y Desarrollo. Vol. $\mathrm{X}, \mathrm{N}^{\circ} 4$ : 71-78.

LAZO, A. 1996. "Impulso del ecoturismo en áreas silvestres protegidas: la inversión más natural", Chile Forestal, Año XXI, $\mathrm{N}^{\circ}$ 237: 34-36.

MANNING, E. 1996. "Turismo: ¿dónde están los límites?", Ambiente y Desarrollo. Vol. XII, $\mathrm{N}^{\circ} 4$ : 70-76.

OTERO, A., H. RIVAS. 1995. "Turismo y medio ambiente (II): Estándares para la sustentabilidad ambiental del sector turismo", Ambiente y Desarrollo. Vol XI, $\mathrm{N}^{\circ} 4$ : $14-18$ 
PINO, M. 1998. Monte Verde: el rastro del primer hombre americano. Diario Austral, Revista Crónicas del Domingo 12 de julio de 1998, p. 6-7.

RAMIREZ, F. 1996. "La necesidad de avanzar hacia una historia ecológica para Chile", Ambiente y Desarrollo. Vol. XII, $\mathrm{N}^{\circ}$ 2: 61-68.

RIVAS, H., P. VILLARROEL. 1995. "Turismo y medio ambiente I: el turismo en espacios naturales como alternativa estratégica de desarrollo regional", Ambiente y Desarrollo, Vol XI, $\mathrm{N}^{\circ}$ 4: 7-13

SANCHEZ, A., G. DONOSO. 1996. "El valor de existencia y los límites de su validez como valor económico", Ambiente $y$ Desarrollo. Vol. XII, $\mathrm{N}^{\circ}$ 2: 33-39.

SEPULVEDA, C. 1997. "Incentivos para la creación y manejo de Areas Silvestres Protegidas Privadas en Chile", Ambiente y Desarrollo. Vol. XIII, $\mathrm{N}^{\circ}$ 3: 38-46.

SEPULVEDA, C., P. VILlARROEL, D. GARCiA. A. MOREIRA. Proyecto FONDECYT N ${ }^{\circ}$ 1961043. Modali- dades de Cooperación público-privados para la conservación de la biodiversidad en Chile. Informe Final Preliminar 1996-1998.

SERNATUR (SERVICIO NACIONAL DEL TURISMO), DEPARTAMENTO DE PLANIFICACION. 1995. El Turismo: Análisis y Estadísticas (1990-1994). Santiago.

TÖREY, S. 1996. "El impacto ambiental del consumo en Chile. Nivel de ingreso y patrones de consumo en Chile", Ambiente $y$ Desarrollo. Vol. XII $\mathrm{N}^{\circ} 2$ : 11.

VEBlEN, T., R. DELMASTRO, J. SCHLATTER. 1976. "The conservation of Fitzroya cupressoides and its environment in Southern-Chile", Environment. Conserv. 3: 291-301.

VEBLEN, T., D. ASHTON. 1982. "The regeneration status of Fitzroya cupressoides in the Cordillera Pelada, Chile", Biolog. Conserv. 23: 141-161.

VILLARROEL, P. 1996. "El caso de Puerto Natales-Torres del Paine, XII Región: efecto del turismo en el desarrollo local", Ambiente y Desarrollo. Vol XII, $\mathrm{N}^{\circ} 4$ : 58-64. 